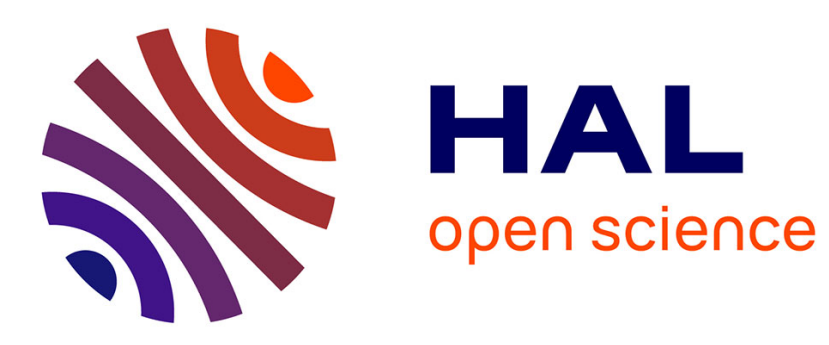

\title{
A mixed GPC-H infinity robust cascade position-pressure control strategy for electropneumatic cylinders
}

Lotfi Chikh, Philippe Poignet, François Pierrot, Cédric Baradat

\section{To cite this version:}

Lotfi Chikh, Philippe Poignet, François Pierrot, Cédric Baradat. A mixed GPC-H infinity robust cascade position-pressure control strategy for electropneumatic cylinders. ICRA: International Conference on Robotics and Automation, May 2010, Anchorage, United States. pp.5147-5154, 10.1109/ROBOT.2010.5509386 . hal-00466773

\section{HAL Id: hal-00466773 \\ https://hal.science/hal-00466773}

Submitted on 25 Mar 2010

HAL is a multi-disciplinary open access archive for the deposit and dissemination of scientific research documents, whether they are published or not. The documents may come from teaching and research institutions in France or abroad, or from public or private research centers.
L'archive ouverte pluridisciplinaire HAL, est destinée au dépôt et à la diffusion de documents scientifiques de niveau recherche, publiés ou non, émanant des établissements d'enseignement et de recherche français ou étrangers, des laboratoires publics ou privés. 


\title{
A mixed GPC- $\mathcal{H}_{\infty}$ robust cascade position-pressure control strategy for electropneumatic cylinders
}

\author{
Lotfi Chikh ${ }^{\dagger}$, Philippe Poignet ${ }^{\ddagger}$, François Pierrot ${ }^{\ddagger}$ and Cédric Baradat ${ }^{\dagger}$
}

\begin{abstract}
A robust cascade strategy combining an outer position predictive control loop and an inner $\mathcal{H}_{\infty}$ pressure control loop is proposed and tested on an electropneumatic testbed for parallel robotic applications. Two types of cylinders are tested, the standard double acting cylinder and the rodless one. A position/pressure difference (or force) strategy is developed and implemented. As the behavior of the nonlinear cylinders is nonlinear, a feedback linearization strategy is adopted. A Generalized Predictive Controller (GPC) is synthesized for the position outer loop and a constrained LMI based $\mathcal{H}_{\infty}$ controller is synthesized for the pressure inner loop. Experimental results show the feasibility of the control strategies and good performances in terms of robustness and dynamic tracking.
\end{abstract}

\section{INTRODUCTION}

The paper is motivated by pick-and-place parallel robotic applications. Within this context, the purpose is to evaluate the position control performances of pneumatic cylinders which are widespread in industry and interesting because of their low cost. Parallel robots have a lot of advantages which have made their success. Particularly, they can be very fast as the actuators are transferred to the base frame reducing considerably the inertia of the moving links. This enables reaching speeds and accelerations which were unbelievable a decade ago. For instance, the fastest industrial robot in the world -the Quattrohas a parallel structure and can reach an acceleration of $15 \mathrm{~g}$ [1]. Recently, the Par2 robot which is not yet industrialized has reached $43 \mathrm{~g}$ while keeping a low tracking error [2]. However, a major obstacle for parallel robot expansion is their expensive price due to the cost of their motors. In this context, considering other types of actuation such as pneumatic actuators is appealing as they are cheap actuators with low maintenance costs, and with a good force/weight ratio. As a major obstacle of industrial use of pneumatic actuation in robotics is the difficulty in their control, this paper focuses on that topic.

Numerous techniques have been studied in literature and among them a large part concerns robust control. Robust controllers are mandatory to deal with disturbances and uncertainties and to ensure high precision positioning. They have been applied as a nonlinear feedback using sliding modes [3] [4] [5] or adaptive control techniques [6], [7] or by using linear robust control techniques such as $\mathcal{H}_{\infty}$ control [8] [9] after feedback linearization [10] [11]. The major drawback

\footnotetext{
$\dagger$ Authors are with Fatronik France Tecnalia, Cap Omega Rond-point B. Franklin, 34960 Montpellier Cedex 2, France. lchikh@fatronik.com and cbaradat@fatronik.com

$¥$ Authors are with the Laboratoire d'Informatique, de Robotique et de Microélectronique de Montpellier (LIRMM). 161 rue Ada, 34392 Montpellier Cedex 5, France. poignet@lirmm.fr and pierrot@lirmm.fr
}

of nonlinear controllers is the difficulty in the synthesis of the control law and the high computational amount. In case of feedback linearization with position as a control output, a zero dynamic exists and there is no global proof of its stability [12].

In this paper, a novel robust cascade strategy which combines an outer position predictive controller and an inner $\mathcal{H}_{\infty}$ pressure controller is proposed. The advantage of the proposed strategy is a simplified control synthesis as only linear robust controllers are implemented. There is no remaining zero dynamic as feedback linearization is applied only for the pressure inner pressure loop. At the same time cascade control enables to make tracking control of two outputs in a SISO (Single Input Single Output) manner. A Generalized Predictive Controller (GPC) is synthesized for the outer loop. As far as we know, only two experimental studies of GPC on pneumatic cylinder have been carried out so far [13] [14]. The model used in [13] is a linear one which limits greatly the performances of the controller. In [14], the authors used a model estimation based on Neural network theory [14]. No application of GPC based on an explicit nonlinear model has been found in literature. In our case, the linearization allows to obtain an explicit solution to the predictive optimization problem and the obtained controller is easy to implement. Another intuitive motivation for using predictive theory in pick-and-place robotic applications is that most trajectories are determined a priori and therefore, future trajectory is known. For the inner pressure loop, a constrained $\mathcal{H}_{\infty}$ controller is developed based on LMI optimization [15] [16]. In addition to the classical advantages of $\mathcal{H}_{\infty}$ control [17] [18] in terms of robustness, disturbance rejection, systematic synthesis of MIMO controllers and powerful combination of both frequency domain synthesis and state space synthesis, the LMI approach enables the addition of constraints in an intuitive manner. Therefore, pole placement constraints have been added to the $\mathcal{H}_{\infty}$ performances in order to have a better control of the transitory temporal behavior of the pressure controller.

This paper is organized as follows. Section II presents the versatile electropneumatic testbed for robotic applications and its nonlinear modeling. Section III deals with the control of the cylinders. After introducing the feedback linearization equations, both of GPC controller background and LMI based $\mathcal{H}_{\infty}$ multi-objective approach are presented. The cascade strategy which combines these two control techniques is then introduced. Finally in section IV, the robust cascade strategy is implemented experimentally and various control tests, including robustness tests, are presented and analyzed. 


\section{EXPERIMENTAl PNeumatiC TESTbed AND NONLINEAR MODELING}

In the long term, our aim is to design efficient parallel robots which rely on pneumatic actuation with robust control: the moving arm of this testbed is very similar to the arms used for various parallel robots such as Delta or Quattro robots[1] which is represented in Fig. 2. At its extremity, different masses can be attached for robustness tests of the controllers as the load varies. The proposed setup shown in Fig. 1 replaces expensive motor-and-gears system by low-cost pneumatic actuator. Three types of actuators are available here. The first one is a standard double acting cylinder ${ }^{1}$ which is very widespread in industry. It is one of the cheapest pneumatic actuators and has been largely studied in literature ([19], [20], [21] and [12]). The second one is the rodless cylinder ${ }^{2}$ which has the advantage of being symmetric, that is to say, the maximum force provided in one direction equals the one in the inverse direction. Another important advantage for the rodless cylinder is that it has generally a larger stroke than the double acting one. Finally, Pneumatic Artificial Muscles ${ }^{3}$ (PAMs) are also installed in the setup but not presented in this paper. It seems that PAMs may be naturally advantaged in rehabilitation comanipulation tasks implying friendly robot designs [22]. It is a common thing to use PAMs in agonist/antagonist way for angle movement generation or torque control. More details about PAMs can be found in [23] [24] and [5].
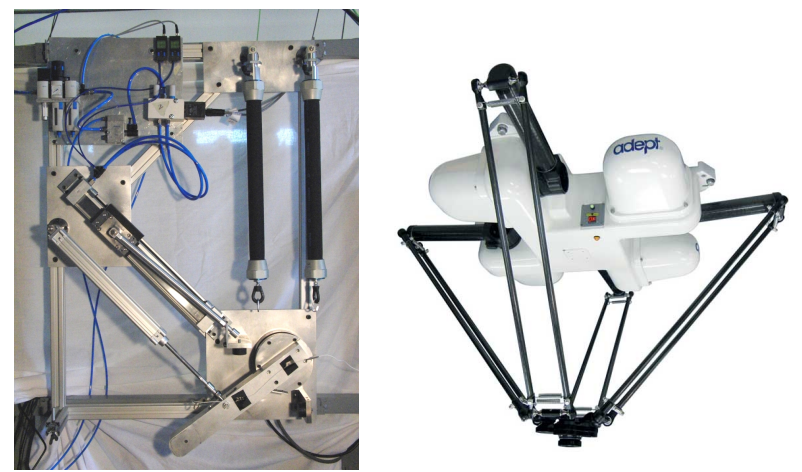

Fig. 1. Versatile electropneumatic Fig. 2. Adept Quattro [1]. setup for robotic application.

All actuators can be driven by two 5/3-way proportional valves $^{4}$. Three types of sensors are used on the setup; a high resolution incremental encoder, two pressure sensors and two force sensors. The real time prototyping environment is xPC Target $^{T M}$ from Mathworks.

In the sequel, we present some key elements of the nonlinear model of the pneumatic cylinders. Every electropneumatic positioning device includes an actuation element (the pneumatic cylinder), a command device (the valve), a mechanical part and position, pressure and/or force sensors. A schematic representation of the electropneumatic system is given in Fig. 3. Supply pressure $p_{s}$ is supposed to be constant. $p_{0}$ denotes

\footnotetext{
${ }^{1}$ Double acting cylinder: FESTO DNC 32320 PPV A

${ }^{2}$ Rodless cylinder: FESTO 532448 DGC 32300304132 ZR

${ }^{3}$ PAMs: FESTO MAS - 20-450N-AA-MC-K

${ }^{4}$ MPYE-5-1/4-010-B from FESTO
}

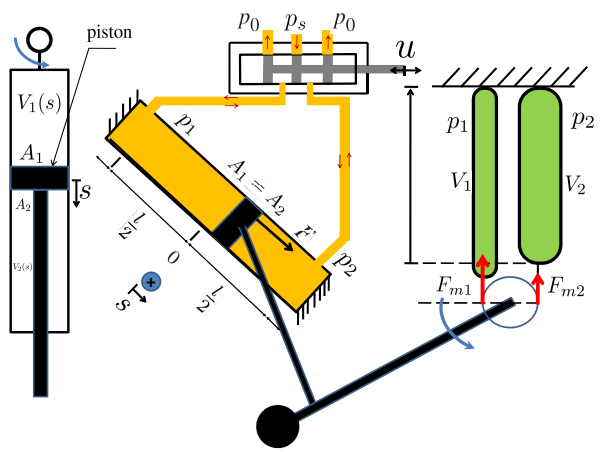

Fig. 3. Schematic representation of the experimental setup

the atmospheric pressure. It is supposed that any variation of the chamber volume or pressure can be described by the polytropic gas law [25]:

$$
p_{1} V_{1}^{\gamma}=p_{2} V_{2}^{\gamma}
$$

where $p_{i}$ is the pressure in one of the two cylinder chambers (indexes 1 and 2 are related to two pressure states) and $V_{i}$ is the volume of one chamber, $\gamma$ is the polytropic constant. The ideal gas equation describes the dependency of the gas mass:

$$
m=\frac{p V}{r T}
$$

where $m$ is the gas mass inside the cylinder chamber, $T$ is the air temperature which is considered to be equal to the atmospheric temperature and $r$ is the specific gas constant. Therefore, combining equation (1) and (2) leads to the pressure dynamic expression:

$$
\frac{d p}{d t}=\frac{\gamma}{V(s)}\left[r T q_{m}(u, p)-p \frac{d V}{d s} \dot{s}\right]
$$

where $u$ represents the input voltage of the valve, $s$ is the position of the piston in case of the cylinders. $q_{m}(u, p)$ represents the mass flow rate $\left(\frac{d m}{d t}=q_{m}(u, p)\right)$. Dynamic effects of the underlying position controller for the valve-slide stroke are neglected. These hypotheses justify why the mass flow is a function of the input voltage and the pressure in the actuator chamber.

The relation between volume and displacement is given by the following equations: $V_{i}(s)=V_{i}(0) \pm A_{i} s$ where $i$ refers to one of the two chambers and $V_{i}(0)=A_{i} \frac{l}{2}$ represents the initial volume. $l$ is the length of the cylinders. $A_{i}$ represents the piston section of each chamber. For the rodless cylinder, sections are symmetric (i.e. $A_{1}=A_{2}$ ). The valve model has been approximated -after identification- by the following expression (see [26] for details):

$$
q_{m}(u, p)=\varphi(p)+\psi(p) u
$$

$\varphi$ and $\psi$ defines $5^{t h}$-order polynomials with respect to $p$.

For the mechanical part, neglecting the friction phenomena and applying Newton's second law lead to:

$$
\ddot{s}=\frac{1}{M}\left[A_{1} p_{1}-A_{2} p_{2}\right]
$$


where $M$ represents the equivalent mass of the moving parts considered at the cylinder side. In the design phase, $M$ is considered as constant.

For cascade control experiments, it is mandatory to determine the force/pressure dependency. This dependency is linear and given by (for standard cylinder):

$$
F_{\text {standard }}=A_{1} p_{1}-A_{2} p_{2}+F_{g}
$$

where $F_{g}$ is the term which takes into account the gravity effects. For the rodless cylinder, we have the same expression but $A_{1}=A_{2}=A$ as we have piston area symmetry.

\section{CONTROL OF THE ELECTROPNEUMATIC CYLINDERS}

In this section, we first present the feedback linearization equations for the cylinders. The approach used here is inspired by differential geometry concepts [10] and differential flatness theory [11]. Once the linearized system obtained, GPC and LMI based constrained $\mathcal{H}_{\infty}$ control techniques are presented.

\section{A. Feedback linearization equations}

It can be easily proved -in case of valve voltage as an input and the pressure difference as an output- that differential flatness criterion is satisfied and that the system is completely linearizable (using differential geometry concepts [10]). However, when position is taken as a control output, the relative degree is less than the dimension of the system. Therefore the system is not completely linearizable. A nonlinear zero dynamic remains. One of the advantages of the cascade strategy is that it avoids dealing with the zero dynamic.

$$
\begin{aligned}
\frac{d p_{1}}{d t} & =\frac{\gamma r T}{V_{1}(s)} \phi\left(p_{1}\right)-\frac{\gamma}{V_{1}(s)} p_{1} \frac{d V_{1}}{d s} \dot{s}+\frac{\gamma r T}{V_{1}(s)} \psi\left(p_{1}\right) u \\
\frac{d p_{2}}{d t} & =\frac{\gamma r T}{V_{2}(s)} \phi\left(p_{2}\right)-\frac{\gamma}{V_{2}(s)} p_{2} \frac{d V_{2}}{d s} \dot{s}-\frac{\gamma r T}{V_{2}(s)} \psi\left(p_{2}\right) u
\end{aligned}
$$

In these equations, we have considered that $u=u_{1}=-u_{2}$ which means that the chambers rely on the same pressure source and use a control signal of an opposite sign. If we do the assumption that the valve is symmetric, this means that only one valve can be used for our control scheme which is in fact an important advantage of this approach. This is represented in Fig. 3 where only one valve is used for each cylinder. By replacing the volume variation by its value (ie, $\left.\frac{d V_{1}}{d s}=A=-\frac{d V_{2}}{d s}\right)$ we get:

$$
\begin{aligned}
\frac{d p_{1}}{d t} & =\frac{\gamma r T}{V_{1}(s)} \phi\left(p_{1}\right)-\frac{\gamma A}{V_{1}(s)} p_{1} \dot{s}+\frac{\gamma r T}{V_{1}(s)} \psi\left(p_{1}\right) u \\
\frac{d p_{2}}{d t} & =\frac{\gamma r T}{V_{2}(s)} \phi\left(p_{2}\right)+\frac{\gamma A}{V_{2}(s)} p_{2} \dot{s}-\frac{\gamma r T}{V_{2}(s)} \psi\left(p_{2}\right) u
\end{aligned}
$$

Pressure difference is given by the following expression:

$$
\begin{aligned}
& \frac{d}{d t}\left(p_{1}-p_{2}\right)=\underbrace{\left[\frac{\gamma r T}{V_{1}} \phi\left(p_{1}\right)-\frac{\gamma r T}{V_{2}} \phi\left(p_{2}\right)-\frac{\gamma A}{V_{1}} p_{1} \dot{s}-\frac{\gamma A}{V_{2}} p_{2} \dot{s}\right]}_{f_{1}\left(s, \dot{s}, p_{1}, p_{2}\right)} \\
& +\underbrace{\left[\frac{\gamma r T}{V_{1}} \psi\left(p_{1}\right)+\frac{\gamma r T}{V_{2}} \psi\left(p_{2}\right)\right]}_{f_{2}\left(s, \dot{s}, p_{1}, p_{2}\right)} u
\end{aligned}
$$

Therefore, the control input which linearizes the input/output behavior of the system is given by:

$$
\begin{aligned}
& u=\left[\frac{\gamma r T}{V_{1}} \psi\left(p_{1}\right)+\frac{\gamma r T}{V_{2}} \psi\left(p_{2}\right)\right]^{-1} \cdot\left[u_{\text {aux }}\right. \\
& \left.-\left(\frac{\gamma r T}{V_{1}} \phi\left(p_{1}\right)-\frac{\gamma r T}{V_{2}} \phi\left(p_{2}\right)-\frac{\gamma A}{V_{1}} p_{1} \dot{s}-\frac{\gamma A}{V_{2}} p_{2} \dot{s}\right)\right]
\end{aligned}
$$

This leads to the first integrator linearized system:

$$
\dot{p}_{1}-\dot{p}_{2}=u_{\text {aux }}
$$

A pressure controller for the first integrator system based on $\mathcal{H}_{\infty}$ theory is then synthesized in the next section.

\section{B. Multi-objective output feedback pressure control via LMI optimization}

An LMI is any constraint of the form:

$$
A(x)=A_{0}+x_{1} A_{1}+\ldots+x_{N} A_{N}<0
$$

where $A_{0} \ldots A_{N}$ are given symmetric matrices and $x^{T}=$ $\left(x_{1} \ldots x_{N}\right)$ is the vector of unknown variables. In (12), the symbol $<$ refers to negative definite ${ }^{5}$.

One way of tuning simultaneously the $\mathcal{H}_{\infty}$ performance and transient behavior is to combine $\mathcal{H}_{\infty}$ and pole placement objectives using LMI optimization techniques. Poles are clustered in regions which can be expressed in terms of LMIs. The class of LMI region defined below has been introduced for the first time by [16]. It turns out to be suitable for LMI-based synthesis.

Def. LMI Regions. A subset $\mathcal{D}$ of the complex plane is called an LMI region if there exists a symmetric matrix $\alpha=\left[\alpha_{k l}\right] \in \mathbf{R}^{m \times m}$ and a matrix $\beta=\left[\beta_{k l}\right] \in \mathbf{R}^{m \times m}$ such that:

$$
D=\left\{z \in \mathcal{C}: f_{\mathcal{D}}(z)<0\right\}
$$

with: $f_{\mathcal{D}}(z):=\alpha+z \beta+\bar{z} \beta_{T}=\left[\alpha_{k l}+\beta_{k l} z+\beta_{l k} \bar{z}\right]_{1 \leq k, l \leq m}$. For instance, we use a disk LMI region centred at $(-q, 0)$ with radius $r$. It is defined below [16]:

$$
f_{\mathcal{D}}(z)=\left[\begin{array}{cc}
-r & q+z \\
q+\bar{z} & -r
\end{array}\right]<0
$$

The constrained $\mathcal{H}_{\infty}$ problem under consideration can be stated as follows [16]. Given an LTI plant:

$$
\begin{aligned}
& \dot{x}(t)=A x(t)+B_{1} \omega(t)+B_{2} u(t) \\
& e(t)=C_{1} x(t)+D_{11} \omega(t)+D_{12} u(t) \\
& y(t)=C_{2} x(t)+D_{21} \omega(t)+D_{22} u(t)
\end{aligned}
$$

an LMI stability region $\mathcal{D}$, and some $\mathcal{H}_{\infty}$ performance $\gamma>0$, find an LTI control law $u=K(s) y$ such that: (1) the closedloop poles lie in $\mathcal{D}$ and (2) $\left\|T_{w e}\right\|_{\infty}<\gamma$ where $\left\|T_{w e}(s)\right\|$ denotes the closed-loop transfer function from $\omega$ to $e$. It is represented on Fig. 4. where the input vector $\omega=\left(\begin{array}{ll}b & v\end{array}\right)^{T}$ can be constituted for instance by a disturbance $b$ and a second input which can be a measure noise $v$. The output vector $e=$ $\left(\begin{array}{ll}y & u\end{array}\right)^{T}$ is composed by the controlled output $y$ and the control signal $u$. In our case, and as defined latter in (39), $y$ is the pressure difference between the chambers and $u$ is the

\footnotetext{
${ }^{5}$ the largest eigenvalue is negative
} 


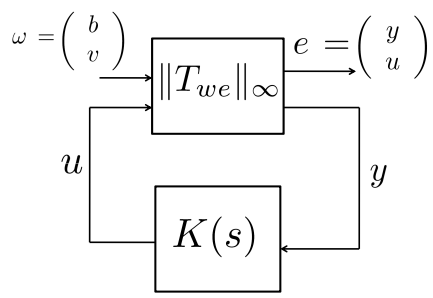

Fig. 4. System representation and notations

input voltage.

The controller transfer function is denoted $K(s)$ and can be represented in the following state-space form by:

$$
\begin{aligned}
& \dot{x}_{K}(t)=A_{K} x_{K}(t)+B_{K} y(t) \\
& u(t)=C_{K} x_{K}(t)+D_{K} y(t)
\end{aligned}
$$

Then, $T_{w e}(s)=D_{c l}+C_{c l}\left(s I-A_{c l}\right)^{-1} B_{c l}$ with $^{6}$ : $A_{c l}:=\quad\left(\begin{array}{cc}A+B_{2} D_{K} C_{2} & B_{2} C_{K} \\ B_{K} C_{2} & A_{K}\end{array}\right), \quad B_{c l} \quad:=$ $\left(\begin{array}{c}B_{1}+B_{2} D_{K} D_{21} \\ B_{K} D_{21}\end{array}\right), C_{c l}:=\left(C_{1}+D_{12} D_{K} C_{2}, D_{12} C_{K}\right)$ and $D_{c l}:=D_{11}+D_{12} D_{K} D_{21}$.

We first examine each specification separately. It is shown in [16] that the pole placement constraint is satisfied if and only if there exists $X_{\mathcal{D}}>0$ such that:

$$
\left[\alpha_{k l} X_{\mathcal{D}}+\beta_{k l} A_{c l} X_{\mathcal{D}}+\beta_{l k} X_{\mathcal{D}} A_{c l}^{T}\right]_{1 \leq k, l \leq m}<0
$$

Meanwhile, the $\mathcal{H}_{\infty}$ constraint is expressed in terms of LMIs:

$$
\left(\begin{array}{ccc}
A_{c l} X_{\infty}+X_{\infty} A_{c l}^{T} & B_{c l} & X_{\infty} C_{c l}^{T} \\
B_{c l}^{T} & -\gamma I & D_{c l}^{T} \\
C_{c l} X_{\infty} & D_{c l} & -\gamma I
\end{array}\right)<0
$$

Then the problem formulation of $\mathcal{H}_{\infty}$ synthesis with pole placement - assuming that the same Lyapunov matrix $X>0$ is required - is:

Find $X>0$ and a controller $K(s)>0 \equiv \Omega_{K}$ that satisfy

(17) and (18) with $X=X_{\mathcal{D}}=X_{\infty}$

The controller matrix is denoted by:

$$
\Omega_{K}=\left(\begin{array}{cc}
A_{K} & B_{K} \\
C_{K} & D_{K}
\end{array}\right)
$$

The difficulty in output feedback is that relations (17) and (18) involve nonlinear terms of the form $\mathcal{B} \Omega_{K} \mathcal{C} X$. This means that problem formulation is not convex and then, can not be handled by LMIs. Chilali and Gahinet [16] solved this problem by taking the following change of variables of the controller:

$$
\begin{aligned}
& \mathcal{B}_{K}:=N B_{K}+S B_{2} D_{K} \\
& \mathcal{C}_{K}:=C_{K} M^{T}+D_{K} C_{2} R \\
& \mathcal{A}_{K}:=N A_{K} M^{T}+N B_{K} C_{2} R+S B 2 C_{K} M^{T} \\
& +S\left(A+B_{2} D_{K} C_{2}\right) R
\end{aligned}
$$

\footnotetext{
${ }^{6}$ we assume that the $D_{22}=0$, this assumption considerably simplifies the formulas. (Note that it is always possible to remove the $D_{22}$ term by a mere change of variables)
}

where $R, S, N$ and $M$ correspond to the following partition of $X$ and it inverse as

$$
\begin{aligned}
& X=\left(\begin{array}{cc}
R & M \\
M^{T} & U
\end{array}\right), X^{-1}=\left(\begin{array}{cc}
S & N \\
N^{T} & V
\end{array}\right) \\
& R \in \mathbf{R}^{n \times n}, S \in \mathbf{R}^{n \times n}
\end{aligned}
$$

The proposed procedure is summarized in the following theorem [16]:

Theorem:

Let $\mathcal{D}$ be an arbitrary LMI region contained in the open left-half plane and let (13) be its characteristic function. Then, the modified problem (19) is solvable if and only if the following system of LMIs is feasible.

Find $R=R^{T} \in \mathbf{R}^{n \times n}, S=S^{T} \in \mathbf{R}^{n \times n}$, and matrices $\mathcal{A}_{K}$, $\mathcal{B}_{K}, \mathcal{C}_{K}$ and $D_{K}$ such that

$$
\begin{gathered}
\left(\begin{array}{cc}
R & I \\
I & S
\end{array}\right)>0 \\
{\left[\alpha_{k l}\left(\begin{array}{cc}
R & I \\
I & S
\end{array}\right)+\beta_{k l} \Phi+\beta_{l k} \phi^{T}\right]_{k, l}<0} \\
{\left[\begin{array}{ll}
\Psi_{11} & \Psi_{21}^{T} \\
\Psi_{21} & \Psi_{22}
\end{array}\right]<0}
\end{gathered}
$$

with the shorthand notation

$$
\Phi:=\left(\begin{array}{cc}
A R+B_{2} C_{K} & A+B_{2} D_{K} C_{2} \\
\mathcal{A}_{K} & S A+\mathcal{B}_{K} C_{2}
\end{array}\right)
$$

$\Psi_{11}, \Psi_{12}$ and $\Psi_{22}$ terms are detailed in appendix B. Given any solution to this LMI system:

- Compute via Singular Values Decomposition (SVD) a full-rank factorization $M N^{T}=I-R S$ of the matrix $I-R S$ ( $M$ and $N$ are then square invertible)

- Solve the system of linear equations (21) for $B_{K}, C_{K}$ and $A_{K}$ (in this order).

- Set $K(s):=D_{K}+C_{K}\left(s I-A_{K}\right)_{-1} B_{K}$.

Then $K(s)$ is an $n^{t h}$ order controller that places the closedloop poles in $\mathcal{D}$ and such that $\left\|T_{w e}\right\|_{\infty}<\gamma$.

\section{GPC outer position Controller}

The GPC algorithm is based on a CARIMA model which is given by:

$$
A\left(z^{-1}\right) y(t)=z^{-d} B\left(z^{-1}\right) u(t-1)+C\left(z^{-1}\right) e(t)
$$

where $u(t)$ and $y(t)$ are respectively the control and output sequences of the plant and $\mathrm{e}(\mathrm{t})$ is a zero-mean white noise. $A, B$ and $C$ are polynomials of the backward shift operator $z^{-1}$. They are given by; $A\left(z^{-1}\right)=1+a_{1} z^{-1}+a_{2} z^{-2}+\ldots+$ $a_{n a} z^{-n a}, B\left(z^{-1}\right)=b_{0}+b_{1} z^{-1}+b_{2} z^{-2}+\ldots+b_{n b} z^{-n b}$ and $C\left(z^{-1}\right)=1+c_{1} z^{-1}+c_{2} z^{-2}+\ldots+c_{n c} z^{-n c}$ and $\triangle=1-z^{-1}$. For simplicity, it is admitted that $C^{-1}$ equals 1. It is important to mention that most SISO plants can be described by a CARIMA model after linearization. The 
GPC algorithm consists in applying a control sequence that minimizes a multistage cost function of the form:

$$
\begin{aligned}
J\left(N_{1}, N_{2}, N_{u}\right)= & \sum_{j=N_{1}}^{N_{2}} \delta(j)[\hat{y}(t+j \mid t)-w(t+j)]^{2} \\
& +\sum_{j=1}^{N_{2}} \lambda(j)[\triangle u(t+j-1)]^{2}
\end{aligned}
$$

where $\hat{y}(t+j \mid t)$ is an optimum $\mathrm{j}$ step ahead prediction of the system output on data up to time $t, N_{1}$ and $N_{2}$ are the minimum and maximum cost horizons, $N_{u}$ is the control horizon, $\delta(j)$ and $\lambda(j)$ are weighting sequences and $w(t+j)$ is the future reference trajectory.

The minimization of cost function leads to a future control sequence $u(t), u(t+1), \ldots$ where the output $y(t+j)$ is close to $w(t+j)$. Therefore, in order to optimize cost function, the best optimal prediction of $y\left(t+j\right.$ ) (for $N_{1} \leq j \leq N_{2}$ ) has to be determined. This needs the introduction of the following Diophantine equation:

$$
1=A_{j}\left(z^{-1}\right) \tilde{A}\left(z^{-1}\right)+z^{-j} F_{j}\left(z^{-1}\right)
$$

with $\tilde{A}=\triangle A\left(z^{-1}\right)$ and polynomials $E_{j}$ and $F_{j}$ are uniquely defined with degrees $j-1$ and $n a$ respectively. $\triangle$ is defined as $\triangle=1-z^{-1}$

By multiplying (27) by $\triangle E_{j}\left(z^{-1}\right) z^{j}$ and considering (29), we obtain:

$$
\begin{aligned}
y(t+j)= & F_{j}\left(z^{-1}\right) y(t)+E_{j}\left(z^{-1}\right) B\left(z^{-1}\right) \triangle u(t+j-d-1) \\
& +E_{j}\left(z^{-1}\right) e(t+j)
\end{aligned}
$$

Since the noise terms in (30) are all in the future (this is because degree of polynomial $E_{j}\left(z^{-1}\right)=j-1$ ), the best prediction of $y(t+j)$ is:

$$
\hat{y}(t+j \mid t)=G_{j}\left(z^{-1}\right) \triangle u(t+j-d-1)+F_{j}\left(z^{-1}\right) y(t)
$$

where $G_{j}\left(z^{-1}\right)=E_{j}\left(z^{-1}\right) B\left(z^{-1}\right)$

Polynomials $E_{j}$ and $F_{j}$ can merely be obtained recursively (demonstration can be found for instance in [27]).

In the future, it will be referred only to $N=N_{2}=N_{u}$ as the prediction horizon. $N_{1}$ is chosen equal to 0 .

Let's consider the following set of $j$ ahead optimal predictions:

$$
\begin{aligned}
& \hat{y}(t+d+1 \mid t)=G_{d+1} \triangle u(t)+F_{d+1} y(t) \\
& \vdots \\
& \hat{y}(t+d+N \mid t)=G_{d+N} \triangle u(t+N-1)+F_{d+N} y(t)
\end{aligned}
$$

It can be written in the following compact form:

$$
\mathbf{y}=\mathbf{G} \mathbf{u}+\mathbf{F}\left(z^{-1}\right) y(t)+\mathbf{G}^{\prime}\left(z^{-1}\right) \triangle u(t-1)
$$

where terms $\mathbf{y}, \mathbf{u}, \mathbf{G}^{\prime}$ and $F$ can be defined in [28].

Equation (33) can be rewritten in this form:

$$
\mathbf{y}=\mathbf{G u}+\mathbf{f}
$$

Where $\mathbf{f}$ refers to the last two terms in Eq. (33) which only depend on the past. Now, we are able to rewrite (28) as:

$$
J=(\mathbf{G u}+\mathbf{f}-\mathbf{w})^{T}(\mathbf{G u}+\mathbf{f}-\mathbf{w})+\lambda u^{T} u
$$

where $\mathbf{w}=[w(t+d+1), w(t+d+1) \ldots w(t+d+N)]^{T}$ equation (35) can be written as:

$$
J=\frac{1}{2} \mathbf{u}^{\mathbf{T}} \mathbf{H u}+\mathbf{b}^{\mathbf{T}} \mathbf{u}+f_{0}
$$

with $\mathbf{H}=\mathbf{2}\left(\mathbf{G}^{\mathbf{T}} \mathbf{G}+\lambda \mathbf{I}\right), \mathbf{b}^{\mathbf{T}}=\mathbf{2}(\mathbf{f}-\mathbf{w})^{\mathbf{T}} \mathbf{G}$ and $f_{0}=$ $(\mathbf{f}-\mathbf{w})^{\mathbf{T}}(\mathbf{f}-\mathbf{w})$

Therefore, the minimum of $J$ can simply be found by making the gradient of $J$ equal to zero, which leads to:

$$
\mathbf{u}=-\mathbf{H}^{-\mathbf{1}} \mathbf{b}=\left(\mathbf{G}^{\mathbf{T}} \mathbf{G}+\lambda \mathbf{I}\right)^{-\mathbf{1}} \mathbf{G}^{\mathbf{T}}(\mathbf{w}-\mathbf{f})
$$

Since the control signal that is actually sent to the process is the first element of vector $\mathbf{u}$ (receding strategy), it is given by:

$$
\triangle u(t)=\mathbf{K}(\mathbf{w}-\mathbf{f})
$$

where $\mathbf{K}$ represents the first element of matrix $\left(\mathbf{G}^{\mathbf{T}} \mathbf{G}+\right.$ $\lambda \mathbf{I})^{-1} \mathbf{G}^{\mathbf{T}}$. Contrary to conventional controllers, predictive ones depend only on future errors and not past ones.

D. Position/pressure control strategy for the pneumatic cylinders

In this part, the cascade control concept is presented for the cylinders. The complete control scheme for this concept is summarized in Fig. 5. The inner loop consists in the control of the pressure difference between the two chambers of each cylinder. An LMI based $\mathcal{H}_{\infty}$ controller is implemented. The outer loop consists in the position generalized predictive controller which is implemented based on the closed loop transfer function of pressure difference in cascade with the mechanical dynamics. As presented before, the linearized system in case of pressure difference is an integrator. Therefore, equations of the system can be stated as follows:

$$
\begin{aligned}
& \dot{x}=(0) x+\left(\begin{array}{ll}
1 & 0
\end{array}\right)\left(\begin{array}{l}
b \\
v
\end{array}\right)+(1) u \\
& \left(\begin{array}{l}
y \\
u
\end{array}\right)=\left(\begin{array}{c}
-1 \\
0
\end{array}\right) x+\left(\begin{array}{ll}
1 & 0 \\
0 & 0
\end{array}\right)\left(\begin{array}{l}
b \\
v
\end{array}\right)+\left(\begin{array}{l}
0 \\
1
\end{array}\right) u \\
& y=(1) x+\left(\begin{array}{ll}
0 & 1
\end{array}\right)\left(\begin{array}{l}
b \\
v
\end{array}\right)+(0) u
\end{aligned}
$$

where $\omega=\left(\begin{array}{ll}b & v\end{array}\right)^{T}$ and $e=\left(\begin{array}{ll}y & u\end{array}\right)^{T}$ have been defined before. Classical assumptions for solving $\mathcal{H}_{\infty}$ problem can easily be verified $^{7}$. The result of the LMI optimization is a controller which has the following form:

$$
K(p)=\frac{K_{1} p+K_{2}}{p+K_{3}}
$$

Therefore, the equivalent closed loop pressure transfer function is:

$$
G_{c l_{\text {pressure }}}(p)=\frac{K_{1} p+K_{2}}{p^{2}+\left(K_{1}+K_{3}\right) p+K_{2}}
$$

Based on this transfer function, it is possible to reconstruct the position by integrating the force two times and dividing by the moving mass (5). The GPC controller will be then synthesized using the following transfer function:

$G_{\text {position }}(p)=\frac{K_{1} p+K_{2}}{M p^{2}\left(p^{2}+\left(K_{1}+K_{3}\right) p+K_{2}\right)}=\frac{G_{\text {cl }_{\text {pressure }}}}{M p^{2}}$

${ }^{7}\left(A, B_{2}\right)$ controllable and $\left(C_{2}, A\right)$ observable 


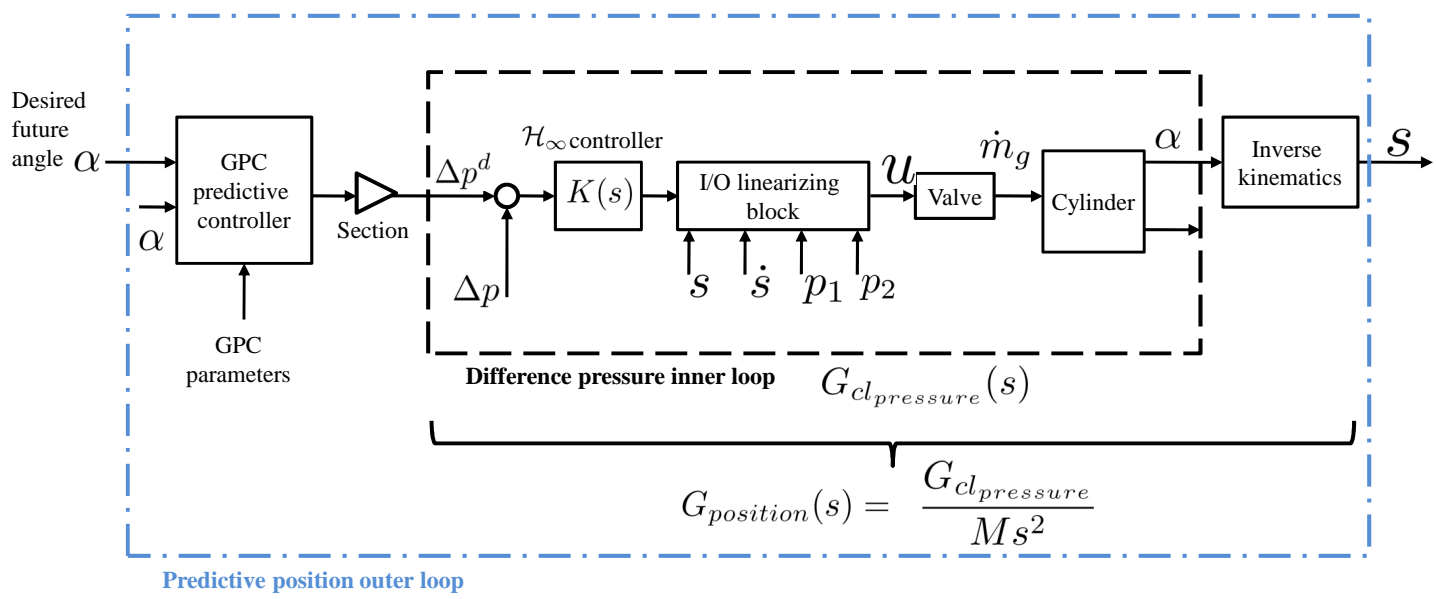

Fig. 5. General block diagram of the cascade Position/pressure strategy for the cylinders. The inner loop consists in the control of the pressure difference between the two chambers of each cylinder. An LMI based $\mathcal{H}_{\infty}$ controller is implemented. The outer loop consists in the position generalized predictive controller which is implemented based on the closed loop transfer function of pressure difference in cascade with the mechanical inverse dynamics.

\section{EXPERIMENTAL RESULTS}

In the following, the proposed cascade $\mathrm{GPC} / \mathcal{H}_{\infty}$ is implemented on the pneumatic cylinders. Some typical tests are handled and commented in details.

\section{A. Some typical control tests}

During all tests concerning cylinders, the gravity term $F_{g}$ in (6) has been neglected. It has been observed that it has not a big impact on performances. In this context, the design of the cascade strategy is simplified and at the same time it guarantees robustness to gravity effects and orientation variations. A second assumption was made in equation (5) where friction has been neglected. In spite of this, it will be shown that one of the advantages of the multiobjective pressure inner controller is that it can be used to remove friction effects on control performances.

The first series of tests concerns the case where there is no load attached at the end of the arm. The control frequency is $0.2 \mathrm{Khz}$. For the $\mathcal{H}_{\infty}$ pressure controller, a disk LMI
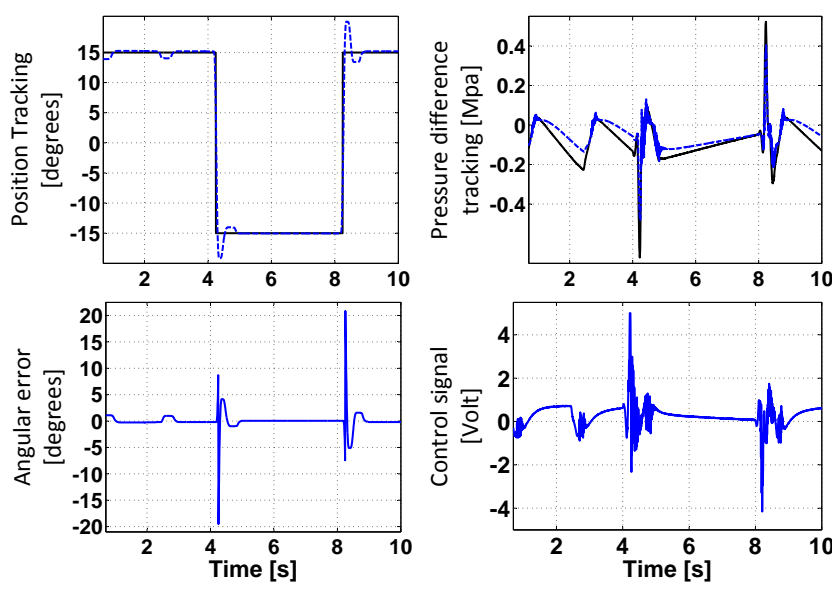

Fig. 6. Pulse response of the rodless cylinder region (see [16] [29]) has been chosen because it enables to avoid fast dynamic poles. We have tried to find a tradeoff between oscillations that appear for fast dynamic poles and time response that will be longer for slow dynamic ones. After some empirical simulations, a disk centered at point $(-300,0)$ with a radius of 15 has been chosen leading to a satisfying behavior. These parameters are used to define the disk LMI region defined by (14).

Using constraints (23), (24) and (25) for the system defined by (39) we obtain after LMI optimization [15] the terms $R$, $S, \mathcal{A}_{K}, \mathcal{B}_{K}, \mathcal{C}_{K}$ and $D_{K}$. We are now able to compute the constrained $\mathcal{H}_{\infty}$ controller which is given by:

$$
K(p)=\frac{-263.8 p-9.15 \cdot 10^{-4}}{p+339.8}
$$

The outer position controller is designed based on the equivalent obtained transfer function given by (42). In order to apply GPC, it is necessary to do the discretization of the transfer function. This is done with a sampling time $T_{s}=5 \mathrm{~ms}$. We obtain:

$$
\begin{gathered}
B\left(z^{-1}\right)=4.63 \cdot 10^{-7}+1.3 \cdot 10^{-6} z^{-1}-2 \cdot 10^{-10} z^{-2}-4.3 \cdot 10^{-3} \\
A\left(z^{-1}\right)=1-2.44^{z^{-1}}+2 z^{-2}-0.54 z^{-3}+0.05 z^{-4}
\end{gathered}
$$

The weighting factor $\lambda$ has been chosen equal to $5 \cdot 10^{-6}$ and $\delta$ is fixed to 1 . The prediction horizon equals 50. On Fig. 6 , a pulse signal reference with an amplitude of $-+15^{\circ}$ is given to the system. Experimental results are very good in terms of static error and time response. The system follows easily the reference. The rising time equals $0.045 \mathrm{~s}$ and settling time equals $0.24 \mathrm{~s}$. Steady state error is less then $0.05^{\circ}$ in the negative case and $0.2^{\circ}$ for the positive one. This is due in part to the fact that gravity effect is not the same for the positive or negative position. As observed at time $t=3 \mathrm{~s}$, there is a small jump -less than $1^{\circ}$ - in the output during the steady state period. We think that this jump is due to the sticking and restarting phenomena which is characteristic to 
electropneumatic systems (see chapter 3 of [12]). In our case, our aim is to reach a precision better than $1^{\circ}$ for carrying loads up to $5 \mathrm{~kg}$ in pick-and-place applications. This is achieved in all the tests presented in this document even in presence of restarting phenomena. However, this problem can be removed by changing the closed loop pole location of the inner pressure controller. For instance, rather than a circle LMI region of radius 25 and centered at point $(-350,0)$, we constrain the poles to be at $(-450,0)$. This is represented on figure 7 .

The consequence can be viewed on Fig. 8. The limit cycle

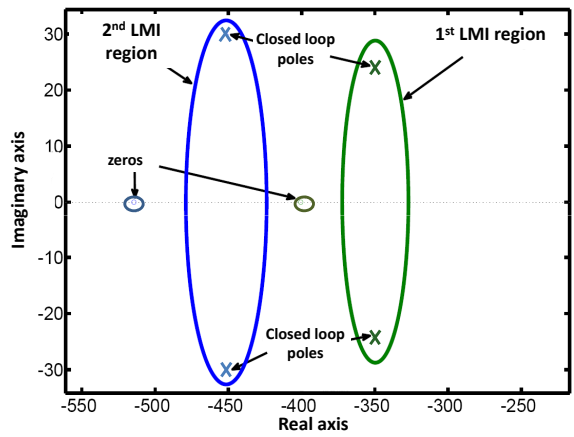

Fig. 7. Zero pole map: LMI circle regions and closed loop pole location

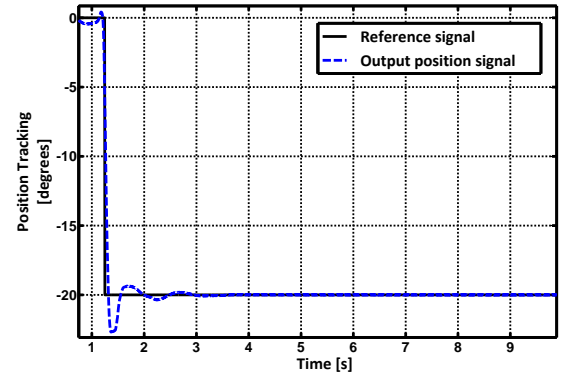

Fig. 8. High gain step response

has been completely removed. The steady state error equals $0.02^{\circ}$.

B. Pressure controller influence and robustness analysis tests: In Fig. 9, a robustness test is done by adding a $5 \mathrm{~kg}$ load at $t=5 \mathrm{~s}$. The regulation is still good and the static error is less than $0.1^{\circ}$. It is interesting to observe that the system reacts by a difference pressure jump which corresponds to the mass variation added at the end of the arm. Therefore, it is possible to deduce that the role of the inner loop is twice; first, it simplifies the control synthesis of the outer controller and secondly it robustifies the control because the difference pressure inner tracking enables a natural adaptation to mass variations.

c. Comparing results with state-of-art former studies: It is not easy to compare those tests with former studies because the experiments and the objectives are not the same. For instance, in [12] [20] [13] [30] [19], the authors present only position control tests without any robustness experiments. In [4], the
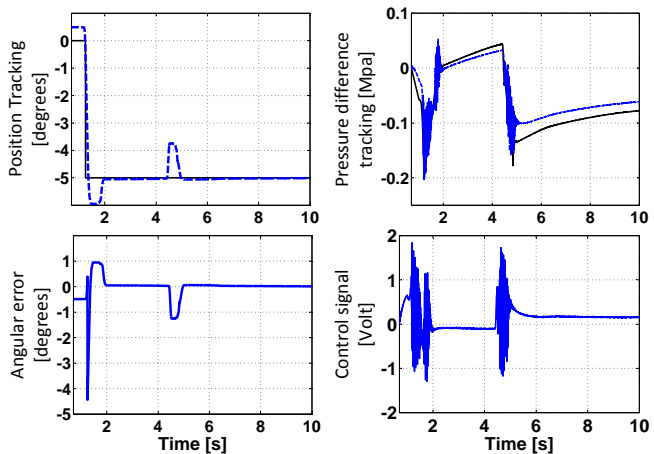

Fig. 9. Robustness test with a $5 \mathrm{~kg}$ mass: a difference pressure jump is observed corresponding to the mass variation. Good regulation with a static error less than $<0.1^{\circ}$.

authors developed a robust sliding mode controller. Robustness is tested by increasing the moving mass by $1.8 \mathrm{~kg}$. Fig. 10 shows optimal test results with the sliding mode controller implemented in [30] and compares chirp signal tracking results with our robust cascade strategy. The experiments have been done with the same control signal amplitudes. This experiment

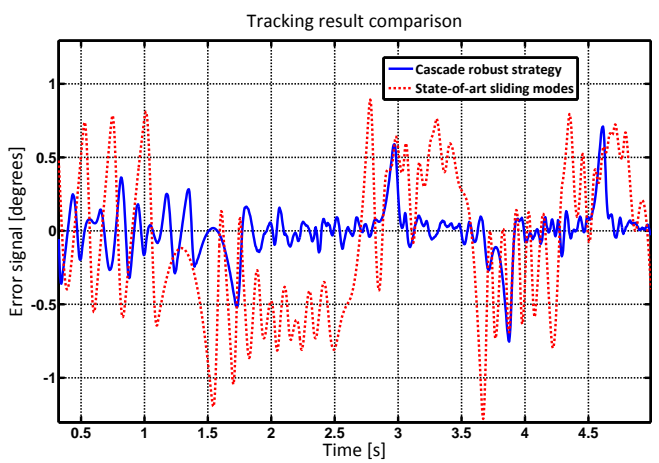

Fig. 10. Experimental chirp signal tracking results: comparison between the robust cascade strategy and state-of-art sliding modes [30]

clearly shows the improvement that can be brought by such a control scheme. In Fig. 11, a robustness test is handled, that is, an unmodeled $4 \mathrm{~kg}$ load is added without considering it in the controller. The gains are the same as for former test. Results show clearly the interest of the cascade robust control strategy introduced here.

\section{CONClusion}

An experimental versatile testbed is designed in order to test the proposed robust cascade strategies on electropneumatic actuators for pick and place parallel robotic applications and based on a mixed GPC and $\mathcal{H}_{\infty}$ approach. As our aim is to reach at least a precision less than $1^{\circ}$ for transporting loads up to $5 \mathrm{~kg}$, the robust cascade strategy fulfills these requirements in spite of the different uncertainties relative to friction, gravity effects and different nonlinearities of the actuators. The different experiments have shown the contribution of the predictive position controller in performances by reducing time delay 


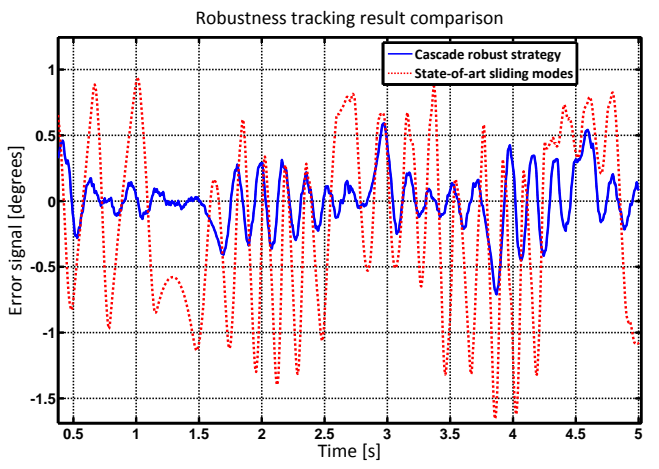

Fig. 11. Experimental $4 \mathrm{~kg}$ mass variation robustness test: tracking results comparison between the robust cascade strategy and state-of-art sliding modes [30]

in a natural manner. Predictive control is particularly suitable for industrial robotics applications where future references are known a priori. On the other hand the different robustness tests have shown the importance of pressure difference inner loop which adapts itself in a natural manner to mass variations. Future work will consist in the improvement of the model by estimating and canceling the friction effects. For the pressure multiobjective controller, the inspection of other LMI region should be interesting in order to remove the chattering effect of the control signal for high dynamic closed loop poles.

\section{ACKNOWLEDGMENT}

The authors would like to thank the Fatronik Tecnalia Foundation for its support with the experimental setup.

\section{REFERENCES}

[1] Adept, http://www.adept.com/.

[2] F. Pierrot, C. Baradat, V. Nabat, S. Krut, and M. Gouttefarde. Above $40 \mathrm{~g}$ acceleration for pick-and-place with a new 2-dof pkm. In IEEE International Conference on Robotics and Automation, May 12 - 17, Kobe, Japan, 2009.

[3] A. Girin, F. Plestan, X. Brun, A. Glumineau, and M. Smaoui. Positionpressure robust control of an electropneumatic actuator. European Control Conference, 2007.

[4] A. Girin, F. Plestan, X. Brun, and A. Glumineau. High-order sliding mode controllers of an electropneumatic actuator: Application to an aeronautic benchmark. IEEE Transactions of Control Systems Technology, 17:633-645, May, 2009.

[5] M. Van Damme, B. Vanderborght, R. Van Ham, B. Verrelst, F. Daerden, and D. Lefeber. Sliding mode control of a 2-dof planar pneumatic manipulator. International Applied Mechanics, 44:1191-1199, 2008.

[6] Xiaocong Zhu, Guoliang Tao, Bin Yao, and Jian Cao. Adaptive robust posture control of a parallel manipulator driven by pneumatic muscles. Automatica, 44(9):2248 - 2257, 2008.

[7] Xiaocong. Zhu, Guoliang. Tao, Bin. Yao, and Jian Cao. Integrated direct/indirect adaptive robust posture trajectory tracking control of a parallel manipulator driven by pneumatic muscles. IEEE Transactions of Control Systems Technology, 17:576-588, 2009.

[8] K. Osuka, Kimura T., and Ono T. H infinity control of a certain nonlinear actuator. In Proceedings of the 29th Conference on Decision and Control. Honolulu, Hawai, December 1990.

[9] T. Kimura, S. Hara, and T. Tomisaka. H infinity control with minor feedback for a pneumatic actuator system. In Proceedings of the 35th Conference on Decision and Control. Kobe, Japan., December 1996.

[10] A. Isidori. Nonlinear Control Systems. Third edition. Communications and Control Engineering Series. Springer-Verlag, Berlin, 1995.
[11] Michel. Fliess, Jean. Lévine, philippe. Martin, and Pierre. Rouchon. Flatness and defect of non-linear systems: introductory theory and examples. International Journal of Control, 61:1327-1361, 1995.

[12] X. Brun. Commandes linéaires et non linéaires en électropneumatique. Méthodologie et applications. PhD thesis, INSA de Lyon, 1999.

[13] Qiang Song and Fang Liu. Improved control of a pneumatic actuator pulsed with pwm. In Proceedings of the 2nd IEEE/ASME International Conference on Mechatronic and Embedded Systems and Applications, 2006.

[14] M. Norgaard, P. H. Sorensen, N. K. Poulsen, O. Ravn, and L. K. Hansen. Intelligent predictive control of nonlinear processes using neural networks. In Intelligent Control, 1996., Proceedings of the 1996 IEEE International Symposium on, pages 301-306, Dearborn, MI, USA, September 1996.

[15] P. Gahinet, A. Nemirovskii, A.J. Laub, and M. Chilali. The Imi control toolbox. In A. Nemirovskii, editor, Proc. 33rd IEEE Conference on Decision and Control, volume 3, pages 2038-2041, 1994.

[16] M. Chilali and P. Gahinet. H-infinity design with pole placement constraints: an lmi approach. IEEE Transactions on Automatic Control, 41(3):358-367, March 1996.

[17] G. Duc and S. Font. Commande Hinf et mu-analyse un outil pour la robustesse. Hermes, 1999.

[18] K. Zhou and J.C Doyle. Essentials of Robust Control. Prentice Hall, 1998.

[19] O. Sawodny and A. Hildebrandt. Aspects of the control of differential pneumatic cylinders. In In. Shimemura and M. Fujita, editors, Proc. of German-Japanese Seminar, pages 247-256., Noto Hanto., 2002.

[20] F. Xiang and J. Wikander. Block-oriented approximate feedback linearization for control of pneumatic actuator system. Control Engineering Practice, 12:387-399, 2004.

[21] A. Ilchmann, O. Sawodny, and S. Trenn. Pneumatic cylinders: Modelling and feedback and feedback force-control. International Journal of Control, 79, Issue 6. June 2006:pages 650 - 661, 2006.

[22] T. D. C. Thanh and K. K. Ahn. Intelligent phase plane switching control of pneumatic artifical muscle manipulators with magneto-rheological brake. International Conference on Control Automation and systems, ICCAS, 2005.

[23] D. Daerden and D. Lefeber. Pneumatic artificial muscles, actuators for robotics and automation. European Journal of Mechanical and Environmental Engineering, 47(1):pages 10 - 21, 2002.

[24] B. Tondu and P. Lopez. Modelling and control of mckibben artificial muscle robot actuators. IEEE Control Systems Magazine, 20:15-38, 2000.

[25] A. Hildebrandt, O. Sawodny, and R. Neumann. A cascaded tracking control concept for pneumatic muscle actuators. In European Control Conference 2003 (ECC03), Cambridge UK, (CD-Rom), 2003.

[26] M. Belgharbi, S. Sesmat, S. Scavarda, and D. Thomasset. Analytical model of the flow stage of a pneumatic servodistributor for simulation and nonlinear control. In The Sixth Scandinavian International Conference on Fluid Power. Tampere-Finlande. Pages 847-860., 1999.

[27] A.E. Camacho and C. Bordons. Model Predicitve Control. Second Edition. Springer, London, 2004.

[28] E.F. Camacho. Constrained generalized predictive control. 38(2):327332, 1993

[29] M. Chilali, P. Gahinet, and P. Apkarian. Robust pole placement in LMI regions. IEEE Transactions on Automatic Control, 44(12):2257-2270, December 1999.

[30] M. Smaoui. Commandes non linéaires robustes mono et multidimensionnelles de dispositifs électropneumatiques: synthèse et applications. $\mathrm{PhD}$ thesis, INSA de Lyon, 2004. 